(Aus dem physiologischen Institut der kgl. Universität in Siena.)

\title{
Die Harnblase als Expulsivorgan. Die glatte Muskelfaser.
}

Von

\section{Prof. B. Boeci.}

(Übersetzt von, Dr. Ph. Verderame, Universitäts-Augenklinik in Turin.)

(Mit 6 Textfiguren.)

\section{Allgemeine Betrachtungen.}

Eine neue Studie über die Harnblase als Ausscheidungsorgan von Flüssigkeit wird demjenigen nicht als unnützes Beginnen erscheinen, der sich mit der einschlägigen Literatur vertraut gemacht und sich überzeugt hat, dass ungleiche und vicht selten sich widersprechende Resultate dieses wichtige Kapitel aus der Physiologie noch unsicher, und daher mit Schwierigkeiten besät, gestalten.

Wenn man auch mit lobenswertem Eifer sowohl in der absteigenden als auch in der aufsteigenden Tierreihe nach Organen und Organteilen mit glatter Muskelfaser gesucht hat, die auf Reize hin mit auf graphischem Wege leicht zu messenden Kontraktionen zu reagieren vermögen, so muss man sich doch eingestehen, dass die Wahl des Organs nicht immer die glucklichste war und dass die Vereinfachung auf dem Wege der Vivisektiion sehr oft auf die äusserste Grenze gebracht worden ist.

Zieht man z. B. in Betracht, dass der Säugetierösophagus auch quergestreifte Muskelfasern aufweist, so sieht man gleich ein, dass die Wahl desselben als Versuchsmaterial weder nützlich noch geeignet sein kann, um die an und für sich schon komplizierten Fragen über die Funktion der glatten Muskelfasern $\mathrm{zu}$ studieren und $z u$ entscheiden; die blosse Überlegung, dass jedwedes hohle Organ mit zwei natürlichen Öffnungen die Ligatur oder den Verschluss einer 
derselben mittelst eines aufblähbaren elastischen Ballons erheischt, genügt schon, um gleich klar zu machen, dass es nicht gerade ratsam sei, den Magen einer. Maus, eines Meerschweinchens, eines Kaninchens, eines Hundes oder einer Katze einer solchen Prozedur zu unterziehen. Ginge man dann so weit, den Parallelismus der glatten, nach einer einzigen Richtung verlaufenden Muskelfasern des Musc. retractor penis des Hundes zu vergessen, um dieses vortreffliche Präparat mit einem - longitudinalen Ösophagusstreifen oder einem transversalen Streifen Froschmagen zu ersetzen, in dem Glauben, im ersteren Falle die residualen inneren Zirkulärfasern und in dem zweiten die äusseren longitudinalen Fasern vernachlässigen zu dürfen, so bildete dies ein eitles und unstatthaftes Beginnen, da dies durch keinen strengen histologischen Befund gestützt wird.

Denn wénn auch letzterer erlaubt, anzunehmen, dass die longitudinale Schicht der glatten Fasern in den mittleren Portionen des Froschmagens sich sehr stark verdünnt, so scheint er doch nicht die Meinung derjenigen $\mathrm{zu}$ bekräftigen, welche die grosse Spärlichkeit solcher Fasern mit deren völligem Fehlen identifizieren; wenn man ausserdem ein solches Präparat mit Hilfe von Tusche mit schwarzen Streifen versieht und mikroskopisch untersucht (Basler), so wird es sich auf einen elektrischen Reiz hin im Sinne seiner grösseren Länge nicht nur verkürzen und verlängern, sondern auch bald nach der einen, bald nach der anderen Seite drehen ${ }^{1}$ ): es beweist dies, dass der Parallelismus der Zirkulärfasern in einem Streifen von nach $\mathrm{Schultz}{ }^{2}$ ) präpariertem Froschmagen weit davon entfernt ist, vollkommen zu sein.

Wenn ich nun heute die experimestelle Behandlung der Funktion der Harnblase wieder aufnehme, so werde ich dazu durch verschiedene Gründe bewogen, welche die Technik betreffen, ferner durch Fragen, die noch nicht gestellt wurden, und Fragen, die man als noch nicht gelöst betrachten kann.

Bei solchen Untersuchungen ist es nicht immer bequem und nützlich, sich des Hundes oder der Katze zu bedienen; die tiefe Narkose, die man oft nicht umgehen kann, verbindert, dass die zu-

1) P. Grützner, Die glatten Muskeln. Ergebn. d. Physiol. Jahrg. 3 Abt. 2. Biophysik und Psychophysik S. 32.

2) P. Schultz, Zur Physiologie der läugsgestreiften (glatten) Muskeln der Wirbeltiere. Arch. f. Phýsiol. 1903 Supplbd. S. 3-4. 
führenden Impulse sich in echte und eigentliche Sensationen umwandeln; daraus ergibt sich der schwerwiegende Nachteil, die reflexwillkürliche Harnblasenkontraktion mit der exquisit reflexen nicht vergleichen zu können.

Die mit einer Bürette bewerkstelligte Einführung von Flüssigkeit in die Harnblase, die dann so leicht ausgestossen wird, zwingt zu einer zweimaligen Ablesung, die schon für sich allein die Aufmerksamkeit des Untersuchers beansprucht.

Die Hebung und die Senkung der Bürette zur manometrischen Ablesung, die auf einer vertikalen Metereinteilung geschieht, bedingen für den Beobachter weitere wichtige Erhebungen; die gebührlich äberwachte Narkose; die Anlegung der Schreibhebel an das Kymoqraphion, die Öffuung und Schliessung des elektrischen Stromes für die Reizung komplizieren die Untersuchungen derartig, dass man Assistenten und Laboratoriumsdiener hinzuziehen muss unter augenscheinlicher Benachteiligung der sonstigen Institutsarbeiten. Dies erklärt, warum die Untersucher das Studium der Harublase in situ als Austreibungsorgan für Flüssigkeiten fast gänzlich verlassen und sich hauptsächlich auf das Studium des entleerten und sorgsam isolierten Organs verlegt haben.

Konnte man dem nicht abhelfen? Dies suchte ich zu erreichen, indem ich die Bürette für die Einführúng der Flüssigkeit in die Harnblase und deren relative Entfernung beibehielt und für die graphische Aufzeichnung der Kontraktionen, statt des Plethysphygmographen nach Mosso, mich des Metallmanometers Marey's bediente. Ein dreifach durchbohrter Hahn, welcher zunächst mit der Bürette und mit dem Manometer bei Aussehluss der Harnblase in Verbindung steht, erlaubte die Ablesung der manometrischen Druckwerte; derselbe Hahn, der im weiteren Verlauf mit der Bürette und mit der Harnblase (bei Ausschluss des Manometers) in Kounmunikation gesetzt wurde, gestattete die bequeme Ablesung der in das Organ gelangten Flüssigkeitsmengen; der gleiche Hahn hot endlich bei dreifacher Kommunikation Gelegenheit, nachzusehen, ob der ursprüngliche Druck ștatiopär blieb (komplete elastische Reaktion) oder vermindert (inkomplete elastische Reaktion) oder endlich vermehrt (hinzugekommene und aufoeschriebene Kontraktion).

'Die Harnblase lässt sich jedoch in ihrer kontraktilen Funktion nicht nur durch Volumsvermehrungen, sondern auch durch geringste Druckunterschiede beeinflussen; wer sich auf die Hebung der Bürette 
rerlässt, bewirkt relativ grosse und unregelmässige, ruckweise Druckschwankuugen. Eine im peripheren Verlauf der zwischen dem Hahn und den benützten Apparaten gelegenen Gummischläuche angebrachte Klemmschraube gestattete eine sehr feine Einstellung der Pression im ganzen System.

Die nicht gestellten Fragen waren vielfache; ich werle auf die bauptsächlichsten von ihuen hinweisen:

Welches war die Elastizilät der exstirpierten Harnblase bei Verlust ihrer Kontraktionsfähigkeit? Welches die Elastizität der exstirpierten, noch elastisch-kontraktilen Harnblase? Welches diejenige der lebenden, durch zirkulierendes Blut versorgte Harnblase? Es ist behauptet worden, dass die Elastizität der letzteren so gross sei, dass zu einer gewissen Zeit Drueke und Volumina in arithmetischer Progression zunähmen. Dies trifft jerloch nicht zu: In einer Phase einfachen Auseinanderfaltens dor schlaffen Wandungen ist die Elastizität fast Null; in einer zweiten Plase ist sie dagegen bedeutend, ohne dass Drucke und Volumina wirklich in arithmetischer Progression zunehmen; in eiuer dritten Phase ist sie unregelmässig, da es unter dem Einfluss des Versuches zu einer Transuldation und Filtration aus den Blasenwandungen kommt. Bei der Harublase gibt es keine Überdehnungsphase mit kontinuierlicher Flüssigkeitsansaugung, wie sie in den elastischen, stark ausclelubaren und dünnen Gummiblasen leicht zur Beobachtung kommen kann.

Soll man vom physiologischen Standpunkt aus beim Meerschweinchen einen inneren Harnblasensphinkter annehmen? Wie unterscheiden sich die leichten rhythmischen Tonusschwankungen der Harnblase von den echten, schwachen Kontraktionen? War die Unterscheidung technisch möglich und physiologisch erkłärbar?

Mosso und Pellacanil) erhielten bei der Hundeharmblase ganz kurze, 6-7 Sek. andauernde, sowie lange (30-40 Sek.) und ganz lange (70-80 Sek.) Kontraktionen. War es nicht gerecht. fertigt und sogar notwendig, nach den Gründen für ein derartiges verschiedenes Verhalten zu suchen? Welcher Art waren vergleichsweise die Wirkungen des Sondierens mit dem Katheter oder mit der Kanüle an der Harnblasenmünòngg, in der muskulösen Urethra und ausserhalb dieser letzteren?

Da bis anhin niemand genaue graphische Darstellungen für die

1) A. Mosso et P. Pellacani, Sur les fonctions de la vessie. Arch. ital. de Biol. t. 1 p. $97-127,291-323.1882$. 
einfach reflexen Harnblasenkontraktionen im Vergleich mit den willkürlich-reflexen gegeben hatte, war es möglich, beide nach Belieben zu erzeugen, unter Erkennung des sie verursachenden, noch unbekannten Mechanismus?

Wenn ja, welches ist die Erklärung für das Harnblasenmyogramm in dem einen und in dem anderen Fall? Wie musste man die Harnblasenretention, die willkürlich-reflexe und die reflexe Miktion erklären? Falls der graphisch-manometrische Apparat Marey's, und dies zum erstenmal, eine so ergiebige Bewertung ler Harnblasenmyogramme gestattet hatte, war dies nicht der beste Beweis dafür, dass der Plethysphygmograph von Mosso wegen seiner grossen Langsamkeit einzig für das Studium der absol ut en Volumwerte reserviert werden sollte, wofür er ja auch eigentlich erdacht worden war?

Unter Benüłzung der abmessbaren, von Bernard ${ }^{\mathbf{1}}$ ) so genau beschriebenen Wirkungen der Curarevergiftung beim Hunde konnten Mosso und Pellacani ${ }^{2}$ ) ohne Bedenken behaupten, dass "la véssie est un esthésiomètre plus sûr que la pression sanguine, et pas inferieur à l'iris même".

Ich habe dagegen gefunden, dass die Harnblase des Meerschweinchens unter mässiger Ausdehnung und Pression von seiten der Flüssigkeit, die sie austreibt und aus den Apparaten zugefübrt erhält, zu regelmässigen, oft auch rhythmischen Aufzeichnungen Veranlassung gibt, die fast unbeeinflusst bleiben von der zeitlichen Kompression der Trachea, von der Reizung durch die Nerven des Zwerchfells, der Muskeln und der Hautsensibilität sowie von der Karotisligatur. Auch nicht in der durch kleine Curaredosen ${ }^{3}$ ) vermehrten Erregharkeitsperiode habe ich mit einiger Sicherbeit Kontraktionen (graphisch registrierbare, also nicht durch einfache Organinspektion festgestellte) bemerken können, sei es, dass ich sie durch Zurufen bewirken wollte, sei es durch Lärm, sei es mit einem leisen Fingerschlag auf den Tisch, sei es durch streichelnde Berührung irgendeiner Körperpartie des Tieres: also durch lauter Mittel, deren sich Mosso mit unfehlbarem Frfolg bedient hatte.

1) Cl. B er nard, Leçons sur la chaleur animale p. 302. Baillière, Paris 1876.

2) A. Mosso et P. Pellacani, Sur les fonctions de la vessie. Arch. ital. de Biol. lib. 1 p. 116-117. 1882.

3) Cl. Bernard, I. c. p. $230-231$. 
Diese Tatsache scheint mir vollkonımen natürlich; denn falls die Harnblase auf die unschädlichsten und flüchtigsten physischen und psychischen Reize hin energisch reagieren müsste, so wärèn Mensch und Tiere durch die fast ununterbrochenen und dringenden Anforderungen eines so undisziplinierten Organs zu einem unerträglichen Sklaventum verurteilt.

\section{Die Elastizität bei den Gummiblasen.}

Trotzdem ich sorgfältige Nachforschungen darüber angestellt habe, ist es mir nicht gelungen, irgendeine Spur einer vergleichenden Studie über das Verhalten der Elastizität in muskulösen und elastischen, hohlen Organen einerseits und in bloss elastischen Blasen anderseits aufzufinden. Angenommen, es gelänge in den ersteren, wie es manchmal in letzteren einzutreten pflegt, wachsende elastische Reaktionen hervorzurufen in demselben oder fast demselben Grad, in welchem die Deformationen wegen des immer mehr zunehmenden Flüssigkeitsgehaltes zunehmen, wer bätte uns sagen können, wann und wie die Muskelkontraktion bei den ersteren vorübergehend oder stetig hinzugetreten wäre und so die Erscheinungen komplizierter gestaltet hätte?

Es war wahrscheinlich, dass es für jedes Hohlorgan ein übertriebenes Nachlassen gäbe, währenddessen die eingeführte mässige Flüssigkeitsmenge eben noch die zusammengefallenen Wandungen zu entfalten vermöchte; in einem solchen Falle wäre die elastische Reaktion oder Pression fast oder ganz Null gewesen. Von diesem Momente an kämen für jede auch noch so kleine Volumzunahme die Ausdehnung und dadurch auch die elastische Reaktion in Betracht; aber hätten dann die Volumina und die Drucke in arithmetischer Progression zugenommen? Parallel dazu, wie bätte sich die Muskelkontraktion erhalten? Welches war für ein gegebenes Hohlorgan die nötige Flüssigkeitsmenge, um die Elastizitätsgrenze zu überwinden? Und kam es nach deren Erreichung zu einer mit Imbibition und Transsudation aus den Wandungen verbundenen Überdehnung?

In einer neueren, im hiesigen Laboratorium ausgeführten Doktoratsthese hatte Buresti ${ }^{1}$ ) in allmählich immer stärker ge-

1) A. Buresti, La funzione del tessuto muscolare liseio negli organi cavi in rapporto con la loro distensione. Tip, Lazzeri, Siena 1907. (B. Bocci Lavori del Labor. di Fisiol. 1904-1909.) 


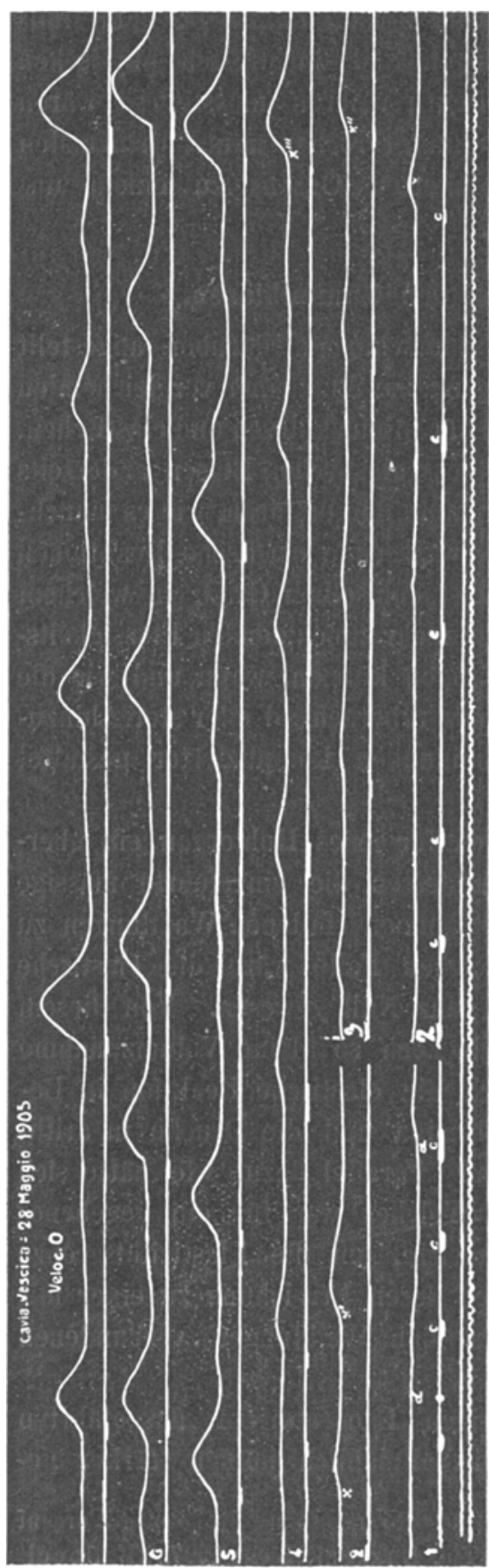

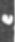

\section{$:=$}

$\dot{n-1}$

dehnten Harnblasen vom Meerschweinchen auf einen elektrischen Reiz hin stärkere Zusammenziehungen erzielt. Als Beleg dafür dient die Fig. 1 (photographisch ungefähr auf ein Drittel der natürlichen Grösse verkleinert): sie zeigt die Zeiteinteilung in Sekunden und in $c c$ auf der Abszisse die Reizungen mit dem Induktionsstrom; $x, x^{\prime}, x^{\prime \prime \prime}$ usw. bezeichnen die mit zunehmender Einführung lauwarmen Wassers, 2, 3, 4, 5, 6, $7 \mathrm{ccm}$, immer stärkeren Harnblasenkontraktionen.

Ähnliche Resultate waren schon von Sokol off und Luehsinger') in den Uretheren erzielt worden. Beobachtungen wie diejenigen von Straub ${ }^{2}$ ), der durch nachfolgende Überdehnungen der Muskulatur des Regenwurmes superponierte Kontraktionen beobachten konnte, werden in der Arbeit $\mathrm{Bu}$ r'esti's nicht erwähnt.

Auf jeden Fall genügen diese Tatsachen sowie die vorhergehenden Betrachtungen

1) O. Sokoloff u. B. Luch. singer, Zur Physiologie der Ureteren. Pflüger's Arch. Bd. 26 S. $464 \mathrm{ff}$.

2) W. Straub, Zur Muskelphysiologie des Regenwurms. Pfluger's Arch. Bd. 79 S. 389. 
zum Beweis dafür, dass es notwerdig 'ist, : vergleichende Untersuchungen über die eigentliche elastische und die elastisch-kontraktile Funktion vorzunehmen, und gerade mit ihuen wollen wir beginnen.

Die Fig. 2 zeigt auf der Ebene $p$ eine dreiwegige, mit einem Hahn versehene Röhre; der Ast $a$ steht in Verbindung mit einer Mohr'schen Bürette, die in Zehntelkubikzentimeter geteilt ist. Der

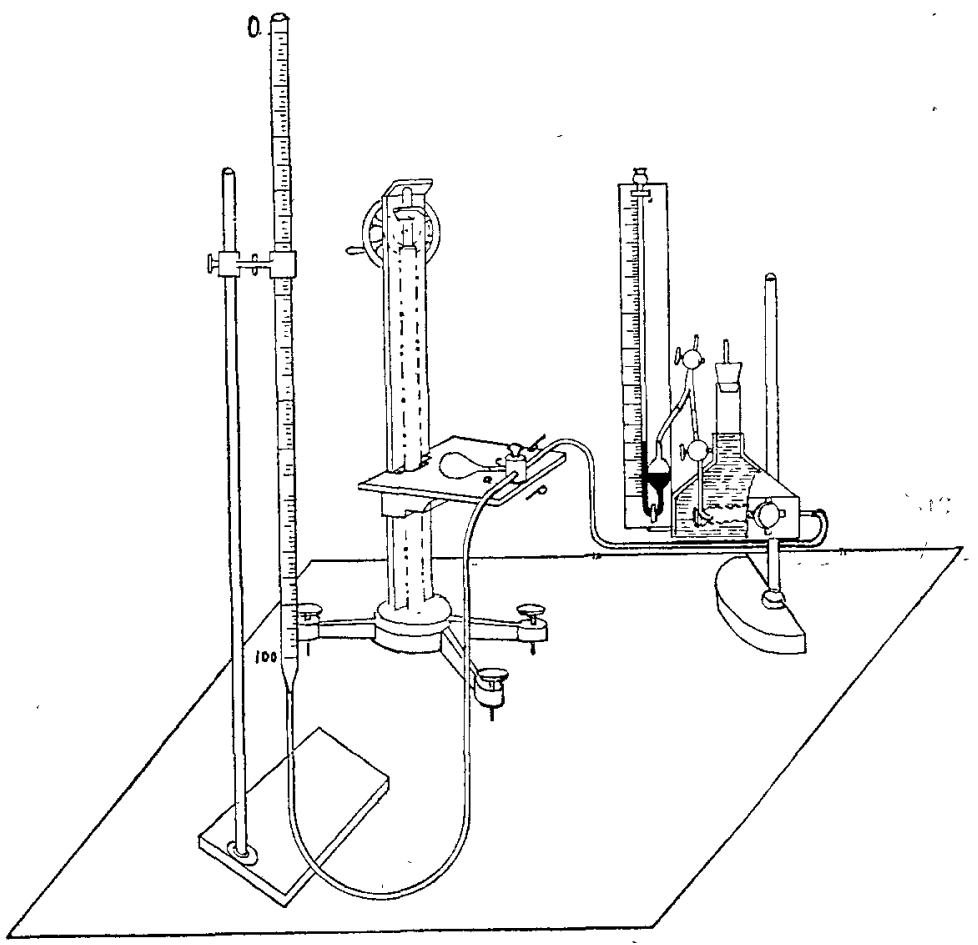

Fig. 2.

gegenüberliegende Ast $b$ steht in Verbindung mit dem metallischen Quecksilbermanometer von M a re y ${ }^{1}$ ); der Ast $c$ endlich kommuniziert vermittelst einer feinen Kanüle mit einem elastischen Gummiballon (Ballonbehälter eines Kondoms). Das zur Verbindung der verschiedenen Teile dienende Kautschukrohr besitzt ungefähr $2 \mathrm{~mm}$ dicke, resistente Wände. Die besonders durch die dreiwegige Röhre gewährleistete gute Funktionierung des Versuchs kann nach vor-

1) E. J. Marey, La méthode graphique dans les sciences expérimentales p. 604-609. Masson, Paris 1878. 
heriger Nivellierung zwischen Manometer und Ebene $p$ sowie nach Fullung des ganzen Systems mit Wasser durch besondere Versuche dargetan werden.

Man bringe den Hahn in die Stellung (1) (Verbindung zwischen Bürette, Blase und Manometer) und suche den Nullpunkt des Druckes. Nun drehe man den Hahn in der Weise, dass der Ballon ausgeschaltet wird (T) und bringe den Manometerdruck auf $10 \mathrm{~mm}$. Es ist klar, dass das Wasser in den Ballon eindringen muss, wenn die Verbindung $z$ wischen ihm und dem Manometer $(\vdash)$ und dann zwischen ihm und der Bürette $(\neg)$ hergestellt wird. Die durch die Flüssigkeit bewirkte Ausdehnung wird die elastische Reaktion und dadurch ein Steigen des Manometerstandes (1) zur Folge haben.

Man gehe, wie vorhin, auf den Druck 0 zurück und dann auf den Druck $10 \mathrm{~mm}$, durch Ausschaltung der Blase. Es leuchtet ein, dass, wenn man den Hahn in der Weise dreht, dass eine Verbindung zwischen der Bürette und dem Gummiballon hergestellt wird, in diesen letzteren Flüssigkeit gelangen wird unter prompter oder langsamer Wiederherstellung des Niveaugleichgewichts in der Bürette. Im ersteren Falle wird der Manometerdruck gar keine oder kaum eine Veränderung erleiden, sofern man die dreifache Verbindung wiederherstellt; im zweiten Falle dagegen wird er eine Veränderung im Sinne der Abnahme erleiden, wodurch die Ablesung beschleunigt wird. Man muss auch bemerken, dass es nicht möglich ist, die letztere stabil zu gestalten, sooft der Gummiballon nach überwundener Elastizitätsgrenze sich weiteraufbläht, bis er endlich platzt.

Danach wird man die zusammenfassenden Resultate der Tab. I leicht verstehen können, aus welcher man eben entnehmen kann, dass die benützte elastische Gummiblase bereits beim Nulldruck durch das eingelassene Wasser leicht auseinandergefaltet wird und dass man ferner durch Senken der Bürette einen negativen Druck bewirken muss, um Wasser in dieselbe zu aspirieren und dessen Menge in zweiter Lesung zu berechnen $(3 \mathrm{ccm})$. Die starke Einziehung und das Zusammenfallen der Blasenwandungen geben den Augenblick an, wo man die oben erwähnte Senkung aussetzen muss. Man kann ausserdem aus der Tabelle entnehmen, dass die Volumina des in die Blase eintretenden Wassers, bei Berücksichtigung einer schwer durchzuführenden gleichmässigen Ablesung der Drucke, wie diese letzteren in arithmetischer Progression zunehmen; dass man 
ferner die Elastizität der Blase als vollkommen annehmen kann und dass endlich, in Betracht der Resistenz ihrer Wandungen, der Höhe der Flüssigkeitssäule in der Bürette und der Grenze ihrer Hebung, es nicht möglich ist, die sogenannte Elastizitätsgrenze zu überschreiten.

Ganz anders verhält sich die Sache, wenn man eine viel dehnbarere und dünnere Gummiblase benützt; ein Blick auf die Tab. II überzeugt uns sogleich davon. Vor allem, sei es, dass die Drucke um je 5 oder um je $10 \mathrm{ccm}$ zunehmen, muss man mit den Versuchen aufhören, sobald der Druck eine gewisse Höhe erreicht hat, indem dann die Blase sich weiteraufbläht. Diese Phase wird durch den Eintritt einer bedeutenden Wassermenge (16,8-15,1 ccm) charakterisiert. Es folgt daraus, dass bei fortgesetztem Sinken des Wassers in der Bürette sich die Ablesung auf das unendliche fortsetzt. Die erhobenen Ablesungen von 16,8-15,1 ccm und die relativen Drucke sind daher sehr weit davon entfernt, strenge zu sein; auch ist es nicht möglich, eine arithmetische Progression der Volumina durchzuführen.

Tabelle I.

Resistente, elastische Gummiblase.

\begin{tabular}{|c|c|c|c|c|}
\hline $\begin{array}{c}\text { Vorbereitende } \\
\text { und nachfolgende Akte }\end{array}$ & $\begin{array}{c}\text { Druck } \\
\text { in mm } \\
\mathrm{Hg}\end{array}$ & $\begin{array}{c}\text { Wasser } \\
\text { in der } \\
\text { Búrette } \\
\text { ccm }\end{array}$ & $\begin{array}{c}\text { Wasser } \\
\text { in der } \\
\text { Gummi- } \\
\text { blase }\end{array}$ & Bemerkungen \\
\hline $\begin{array}{l}\text { Nivellierung und Füllung } \\
\text { des 'ganzen Systems mit } \\
\text { Wasser. Hahu in drei- } \\
\text { facher Kommunikation } \\
\text { und Aufsuchung des Null- } \\
\text { druckes. . . . . . . } \\
\text { Verbindung zwischen Bü- } \\
\text { rette und Gummiblase; } \\
\text { Entleerung der Gummi- } \\
\text { blase. . . . . . - } \\
\text { Wiederherstellung der drei- } \\
\text { fachen Kommunikation } \\
\text { und des Nullpunktes. . } \\
\text { Verbindung zwischen Bü- } \\
\text { rette und Manometer. . } \\
\text { Verbindung zwischen Bú- } \\
\text { rette und Gummiblase. }\end{array}$ & $\begin{array}{c}5 \\
-\frac{10}{-} \\
\frac{15}{-}\end{array}$ & $\begin{array}{l}47,6 \\
48,0 \\
\\
48,1 \\
48,5 \\
48,55 \\
48,9 \\
49,0\end{array}$ & $\begin{array}{l}0,1 \\
\overrightarrow{0,05} \\
\overrightarrow{0,1}\end{array}$ & $\begin{array}{l}\text { Angewandte Zeit für jede } \\
\text { zweimalige Ablesung } \\
\text { (Manometer, Bürette) } \\
1 \text { Min. } \\
\text { Temperatur des Wassers } \\
12^{\circ} \text { C. Unveränderlich- } \\
\text { keit der Drucke in den } \\
\text { ausgeführten Kontroll- } \\
\text { versuchen durch Wieder- } \\
\text { herstellung derdreifachen } \\
\text { Kommunikation zwischen } \\
\text { dem einen und dem an- } \\
\text { deren Sinken des Was- } \\
\text { sers in der Gummiblase. }\end{array}$ \\
\hline
\end{tabular}




\begin{tabular}{|c|c|c|c|c|}
\hline $\begin{array}{c}\text { Vorbereitende } \\
\text { und nachfolgende Akte }\end{array}$ & $\begin{array}{c}\text { Druck } \\
\text { in } \mathrm{mm} \\
\mathrm{Hg}\end{array}$ & $\begin{array}{c}\text { Wasser } \\
\text { in der } \\
\text { Burette } \\
\text { ccm }\end{array}$ & $\begin{array}{c}\text { Wasser } \\
\text { in der } \\
\text { Gummi- } \\
\text { blase }\end{array}$ & Bemerkungen \\
\hline $\begin{array}{l}\text { Wiederherstellung der drei- } \\
\text { fachen Kommunikation } \\
\text { und des Nulldruckes. } \\
\text { Verbindung zwischen Bü- } \\
\text { rette und Manometer. } \\
\text { Verbindung zwischen Bu- } \\
\text { rette und Gummiblase. }\end{array}$ & $\begin{array}{l}\frac{20}{25} \\
\frac{-5}{30} \\
\frac{-}{35} \\
\frac{40}{-}\end{array}$ & $\begin{array}{l}49,4 \\
49,5 \\
49,9 \\
49,95 \\
50,4 \\
50,5 \\
50,95 \\
51,0 \\
54,05 \\
54,15\end{array}$ & $\begin{array}{l}\overline{0,1} \\
\overline{0,05} \\
\overline{0,1} \\
\overline{0,05} \\
\overline{0,1}\end{array}$ & $\begin{array}{l}\text { Bei der Handhabung des } \\
\text { Hahns ist etwas Wasser } \\
\text { ahgeflossen. }\end{array}$ \\
\hline
\end{tabular}

Tabelle II.

Sehr stark dehnbare, elastische Gummiblase.

\begin{tabular}{|c|c|c|c|c|}
\hline $\begin{array}{l}\text { Vorbereitende } \\
\text { und nachfolgende Akte }\end{array}$ & $\begin{array}{c}\text { Druck } \\
\text { in mm } \\
\mathrm{Hg}\end{array}$ & $\begin{array}{l}\text { Wasser } \\
\text { in der } \\
\text { Bürette } \\
\text { ccm }\end{array}$ & $\begin{array}{l}\text { Wasser } \\
\text { in der } \\
\text { Gummi- } \\
\text { blase }\end{array}$ & Bemerkungen \\
\hline $\begin{array}{l}\text { Nivellierung und Fullung } \\
\text { des ganzen Systems mit } \\
\text { Wasser. Hahn in drei- } \\
\text { facher Kommunikation } \\
\text { und Aufsuchung des Null- } \\
\text { druckes. . . . . . . }\end{array}$ & 0 & 54,9 & ? & Wassertemperatur $12^{\circ} \mathrm{C}$. \\
\hline $\begin{array}{l}\text { Kommunikation zwischen } \\
\text { Bürette und Gummiblase; } \\
\text { Entleerung der Gummi- } \\
\text { blase.......... }\end{array}$ & negat. & 51,9 & - & \\
\hline $\begin{array}{l}\text { Wiederherstellung der drei- } \\
\text { fachen Verbindung und } \\
\text { des Nulldruckes. }\end{array}$ & 0 & 54,9 & 3,0 & \\
\hline $\begin{array}{l}\text { Kommunikation zwischen } \\
\text { Bürette und Manometer }\end{array}$ & 5 & 55,3 & - & \\
\hline $\begin{array}{l}\text { Kommunikation zwischen } \\
\text { Bürette und Gummiblase }\end{array}$ & - & 55,6 & 0,3 & \\
\hline
\end{tabular}


Die Harnblase als Expulsivorgan. Die glatte Muskelfaser.

\begin{tabular}{|c|c|c|c|c|}
\hline $\begin{array}{c}\text { Vorbereitende } \\
\text { und nachfolgende Akte }\end{array}$ & $\begin{array}{c}\text { Druck } \\
\text { in } \mathrm{mm} \\
\mathrm{Hg}\end{array}$ & $\begin{array}{c}\text { Wasser } \\
\text { in der } \\
\text { Bürette } \\
\text { ccm }\end{array}$ & $\begin{array}{c}\text { Wasser } \\
\text { in der } \\
\text { Gummi- } \\
\text { blase }\end{array}$ & Bemerkungen \\
\hline $\begin{array}{l}\text { Aufsuchung des Nulldruckes } \\
\text { Verbindung zwischen Bü- } \\
\text { rette und Manometer. - } \\
\text { Verbindung zwischen Bü- } \\
\text { rette und Gummiblase. }\end{array}$ & $\begin{array}{c}\frac{10}{15} \\
\frac{20}{25} \\
\frac{25}{30} \\
\frac{10}{10}\end{array}$ & $\begin{array}{l}56,0 \\
56,4 \\
56,9 \\
57,4 \\
57,8 \\
58,7 \\
59,1 \\
61,2 \\
61,6 \\
78,4 \\
55,7\end{array}$ & $\begin{array}{c}\overline{0,4} \\
\overline{0,5} \\
\overline{0,9} \\
\overrightarrow{2,1} \\
\overrightarrow{16,8} \\
- \\
-\end{array}$ & $\begin{array}{l}\text { Beschleunigte Ablesung; be- } \\
\text { dentende Druckabnahme } \\
\text { (dreifache Kommunika- } \\
\text { tion). }\end{array}$ \\
\hline
\end{tabular}

Dem konstanten Unterschiede von 5 oder 10 in den beiden Druckserien entsprechen in beiden Fällen veränderliche Volumunterschiede.

Wir übergehen viele andere Resultate, da sie zu diesen oder jenen gehören, die unmittelbar vorhergehen (Tab. I). Zusammenfassend muss man beim Studium der Elastizität solcher Gummiblasen folgende Phasen unterscheiden:

$\mathrm{Ph}$ ase der A useinanderfalt ung, bei welcher die zusammengefallenen Wände durch das bald in grösserer, bald in geringerer Menge eindringende Wasser auseinandergefaltet werden. Sie entspricht dem manometrischen Nullpunkte.

Phaseder Distension und derelastischen Reaktion. Wenn der Eintritt der letzteren genau von demjenigen jener abhängt, nehmen Drucke und Volumina in arithmetischer Reihe $z u$; nach der in relativ kurzer Zeit (in ungefähr 1 Minute) vorgenommenen Ablesung am Manometer und darauf in der Bürette ändert sich das Manometerniveau nicht, sofern man durch eine geeignete Drehung des Hahnes die dreifache Verbindung herstellt. Der untersuchte Körper (die Gummiblase in der Tab. I) ist im wahren Sinne des 
Wortes elastisch. Wenn dagegen das Verhalten der elastischen Reaktion nicht genau von der wegen der enthaltenen Flüssigkeit eintretenden Deformation (Distension) abhängt, dann gelingt es nicht, mit den erhaltenen Volumina eine Zahlenreihe zusammenzustellen, welche einen konstanten Unterschied zwischen den einzelnen Zahlen und der vorhergehenden aufweist: wenn man die Ablesung zuerst am Manometer und nachher an der Bürette vornimmt, erleidet das Manometerniveau keine Veränderung oder bloss dann, wenn man durch Drehung des Hahns die dreifache Kommunikation herstellt. Es tritt keine Veränderung ein, wenn die genaue Ablesung an der Bürette in einer relativ kurzen Zeit möglich war; wenn man jedoch die Ablesung wegen des wenn auch geringen, aber doch fortwährenden Sinkens der Flüssigkeit verlängern musste und gleichwohl eine Ausrechnung der Kubikzentimeter vorzunehmen latte, dann sinkt das Manometer, wenn man durch Drehung des Ilahns die dreifache Verbindung herstellt. Der untersuchte Körper (die Blase in der Tab. II) ist unvollständig elastisch.

Phase der Überdehnung. Es handelt sich dabei um die Übertreibung des letzten Phänomens; und in der Tat wird sie von einer sehr verlängerten Ablesung des Büretten- und Manometerniveaus begleitet.

Es muss bemerkt werden, dass die sehr stark dehnbaren und dünnen Gummiblasen leicht platzen.

\section{Die Elastizität in exstirpierten. Harnblasen.}

Bei Anwendung der oben auseinandergesetzten Methode gelangt das Wasser jedesmal, wenn der Hahn von der zwischen Bürette und Manometer bestehenden doppelten Verbindung, durch welche ein positiver Druck erzeugt worden ist, in Verbindung mit der Bürette und der vorher entleerten Harnblase tritt, unfeblbar in die letztere ein, sofern der Katheter bis zum Harnblaseneingang vorgeschoben worden ist und passive oder aktive Zusammenziehungen des Organs es nicht teilweise oder gänzlich verhindern.

Die passiven Kontraktionen entstehen, wenn die volle Blase bei geschlossener oder ganz wenig offener Abdominalhöhle in situ gelassen wird; sie sind meist auf zu ausgiebige Bewegungen des Zwerchfells nach unten hin zurückzuführen, wodurch die Eingeweidemassen und die benachbarten Organe nach unten gedrängt werden. Die aktiven Kontraktionen sind auch in isolierten und exstirpierten 
Blasen gut zu beobachten und sind unter dem Namen von Spontankontraktionen bekannt.

Wenn es auch wahr ist, dass die tiefe Narkose (Chloroform, Äther, Chloral, Morphium, intravenös injizierte Opiumtinktur) das Tier inmobilisiert und zu einer gewissen Zeit die Atmungsbewegungen des Zwerchfells vermindert, so steht es anderseits auch fest, dass diese auch nicht nach Durchschneidung der Zwerchfellnerven gänzlich aufhören; die Lunge bewirkt nämlich durch ihre Auf- und Abblähung, dass das gelähmte Zwerchfell kleine Schwankuugen ausführt, welche mit Hilfe eines kleinen Phrenographen auf einen Schreibhebel übertragen werden können. Die durch einen starken Induktionsstrom bewirkte gleichzeitige Reizung der peripheren

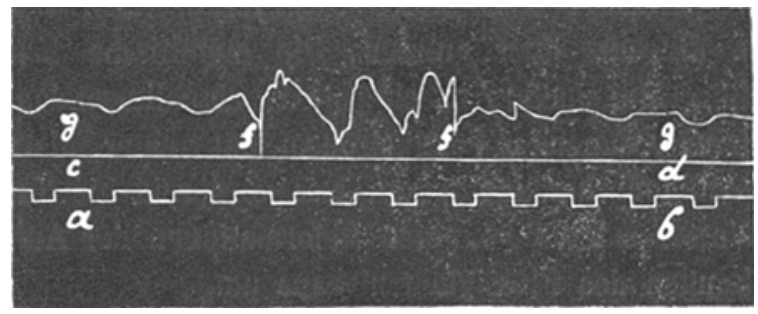

Fig. 3.

Stümpfe der durchsehnittenen Nerven verändert und verstärkt jene kleinen Bewegungen. Die Fig. 3 zeigt dies: $a b$ bedeutet die Zeit in Sekunden ausgedrückt; $c d$ ist die Abszisse; $g f, f g$ Atmungsschwankungen des nach Durchschneiden der Zwerchfellnerven gelähmten Zwerchfells; $f f$ Reizwirkungen. Wenn man derartige kleine Bewegungen vernachlässigt, kann man annehmen, dass die Narkose einerseits und der Schnitt durch die Zwerchfellnerven anderseits in der Mehrzahl der Fälle jede passive Zusammenziehung der Harnblase unmöglich machen; dies ist jedoch nicht in allen Fällen so; denn, wie ich selbst habe beobachten können, die letzten Abschnitte des übermässig gefüllten Darmis und der Uterus bei vorgeschrittener Gravidität vermögen durch Austreibversuche die volle Blase zu beeinflussen, auch wenn die Zwerchfellnerven durehschnitten sind.

Die aktiven Zusammenziehungen der Harnblase kommen durch ihre glatten Muskelfasern zustande, die zu ihrem Bau beitragen; der Name Detrusor muss daher auf die gesamte Muskelwandung 
ausgedehnt werden ${ }^{1}$ ). Wie kann man die elastische Wirkung der Harnblase für sich studieren, wenn man vorher nicht versucht, jene Zusammenziehungen zu verhindern? In dieser Hinsicht ist die Wirkung gewisser Gifte zweifelhaft und unvollständig; man hätte besser seine Zuflucht zur vitalen Frmüdung der Muskelfaser nehmen können, die sicher eintritt infolge unterbundener Blutzirkulation und Aussetzung der Luft bei niedriger Temperatur. Wenn man aber auf diese Weise die kontraktile Funktion in Frage stellt oder gänzlich unterdrückt, wer wird uns dann dafür bürgen, dass nicht gleichzeitig die elastische Funktion Schaden nimmt? Die Lösung der Frage musste in der Paralleluntersuchung isolierter, noch ausgezeichnet kontraktiler und isolierter, auch der geringsten Kontraktion unfähiger Harnblasen gesucht werden.

Man vergewissert- sich dieser entgegengesetzten Bedingungen, indem man die aus dem grossen, die metallische Kammer des Marey'schen Manometers nach oben abschliessenden (Fig. 2) Pfropfen herausschauende Vertikalröhre mit einer Marey'schen Schreibkapsel in Verbindung setzt. Das vorher in die Kammer eingelassene Wasser setzt die inneren Deformationen der Aneroidkapsel in Schwankungen des Flüssigkeitsniveaus um.

Wenn sich jedoch der dreiwegige Hahn in der zur Einführung von Wasser in das Manometer und in die Harnblase geeigneten Stellung befindet, so ist es klar, dass diese Manipulation nicht gleichmässig gestaltet werden kann, solange zur Hebung der Bürette die ungleichmässige und immer verschiedene Bewegung der Hand benützt wird; wenn man in der Tat die Spitze, in welche der Hebel der oben erwähnten Schreibkapsel endigt, einer mit berustem Papier bedeckten rotierenden Trommel nähert, erhält man Kurven entsprechend jenen verschiedenen und unregelmässigen Bewegungen. Die Bürette wurde daher, dieses Mal gefüllt mit wenigen Kubikzentimetern Wassers, an ihrer Stütze stabil fixiert, sobald man den manometrischen Nullpunkt gefunden hatte. Man bewirkte die Druckzunabmen, indem man aus einem aufgesetzten Behälter, welcher mit geeignetem Hahn und mit einem in einer Glasspitze endigenden Gummirobr versehen war, Wasser in die Bürette einfliessen liess.

Fig. 4 zeigt zwei auf diese Weise mit Meerschweinchenharnblasen () erhaltene Aufzeichnungen; man erkennt an ihnen oft den

1) G. Chiarugi, Anatomia dell' nomo lib. 2 fasc. 1 p. 415. 
Zeitpunkt, in welchem das Einfliessen des Wassers beginnt $(\downarrow)$, und wo es aufhört ( $\uparrow$ ). Die Zahlen 10, 20, 30, 40,50 geben in Hg-Millimetern die Anfangsdrucke an vor jeglichen Einfliessens von Wasser. Man darf wohl behaupten, dass die Linien mit grosser Regelmässigkeit und ohne brüske Variationen emporsteigen, ein Beweis dafür, dass die Blasen jede Befähigung zur Kontraktion durch einfache Distension verloren hatten. In der Annahme, dass die stetig zunehmenden Drucke der Muskclreaktion hinderlich sein könnten, unterbrach man sie in anderen Versuchen von Zeit zu Zeit, jedoch immer mit negativem Resultat. Die beiden Harublasen blähten sich gleich nach der Exstirpation etwas mit Wasser auf, das dureh die mit Pean's abgeschlossenen Urethralöffnungen eingelassen wurde, und man liess sie während 36 Stunden der umgebenden Luft ausgesetzt (Temperatur wäbrend des Tages $12^{\circ}$ C., während der Nacht unter $6^{\circ} \mathrm{C}$.).

Es genügt eine aufmerksame Beobachtung der Tab. III und IV, um aus ihr zu entnehmen, dass bei Nulldruck beille Blasen sich unter Wasseraufnahme auseiuanderfalten, dass ferner den Druckzunahmen von je fünf auch versehiedene Volumina entsprechen, dass die zwei charakteristischen Phasen ersichtlich sind (Phase der Auseinanderfaltung, der Ausdehnung und der elastischen Reaktion), dass die elastische Reaktion unvollständig war, dass endlich die Phase der Überdehnung der dünnen Gummi-

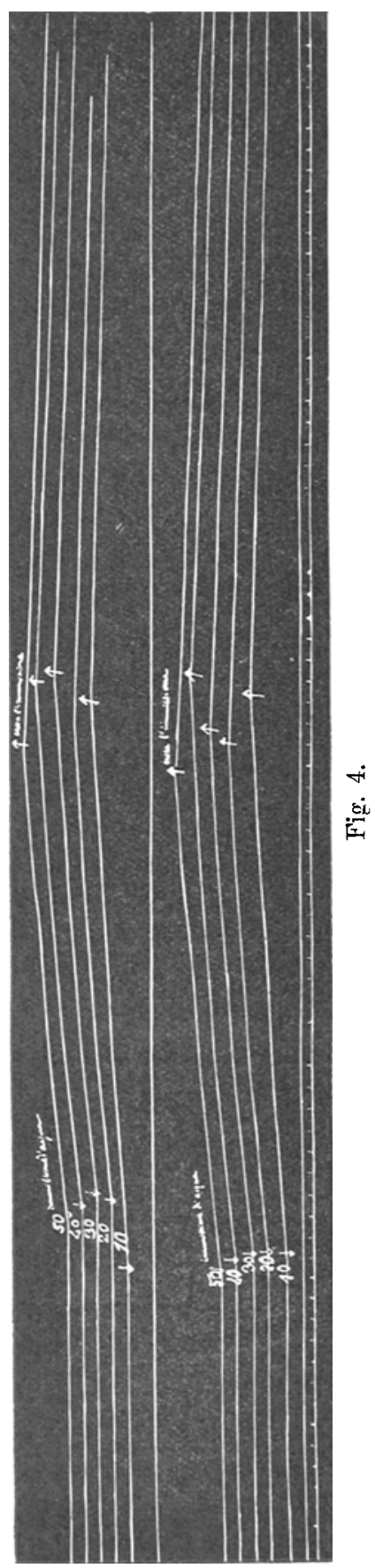


blasen durch die Phase der Imbibition und der Transsudation ersetzt wird, ohne dass für letztere die Anfangsdrucke bei Wiederherstellung der dreifachen Kommunikation des Hahns merklich variieren.

Was würde aus dieser unvollständigen Elastizität der Harnblase werden, wenn man die Untersuchung gleich nach der Entfernung des Organs aus Meerschweinchen vornimmt, die an Verblutung (Durchschneiden der Halsgefässe) eingehen oder enthauptet werden und bei denen man die Sphinkteren und den Muse. detrusor durch Anämie oder Kälte reizt? Die Antwort darauf findet man in den Tab. V und VI, aus denen hervorgeht, dass bei Nulldruck kein Wasser in die Blase hineingelangt und daher die Phase der Auseinanderfaltung ausbleibt; bei einem Drucke von $10 \mathrm{~mm}$ dringen $0,2 \mathrm{ccm}$ Wasser ein, wenn der Katheter den äusseren Sphinkter der Urethra überwunden und über ihn hinaus gedrungen ist, während im entgegengesetzten Fall nichts eindringt, auch nicht bei $30 \mathrm{~mm}$; man kann ausserdem behaupten, dass Volumina und Drucke in arithmetischer Progression zunehmen und dass daher die Phase der Ausdehnung und elastischen Reaktion vollständig ist oder auch, dass sic genau von der durch den Flüssigkeitsgehalt bedingten Deformation abhängt, und dass endlich die letzte Phase der Imbibition und der Transsudation fehlt.

T a belle III.

Exstirpierte Meerschweinchen-Harnblase ( $($ ).

\begin{tabular}{|c|c|c|c|c|}
\hline $\begin{array}{l}\text { Vorbereitende } \\
\text { und nachfolgende Akte }\end{array}$ & $\begin{array}{l}\text { Druck } \\
\text { in } \mathrm{mm} \\
\mathrm{Hg}\end{array}$ & $\begin{array}{c}\text { Wasser } \\
\text { in der } \\
\text { Bürette } \\
\text { cem }\end{array}$ & $\begin{array}{l}\text { Wasser } \\
\text { in der } \\
\text { Blase }\end{array}$ & Bemerkungen \\
\hline $\begin{array}{l}\text { Nivellierung und Füllung } \\
\text { des ganzen Systems mit } \\
\text { Wasser. Hahn in drei- } \\
\text { facher Kommunikation } \\
\text { und Aufsuchung des Null- } \\
\text { druckes. . . . . . }\end{array}$ & 0 & 34,3 & $?$ & $\begin{array}{l}\text { Ungefähre Zeit für jede } \\
\text { zweimalige Ablesung(Ma- } \\
\text { nometer, Bürette) }= \\
1 \text { Min. }\end{array}$ \\
\hline $\begin{array}{l}\text { Kommunikation zwischen } \\
\text { Bürette und Harnblase; } \\
\text { Entleerung der Blase }\end{array}$ & negat. & 33,6 & - & $\begin{array}{l}\text { Wassertemperatur }=12^{\circ} \mathrm{C} \text {. } \\
\text { Druck in den Kontroll- } \\
\text { versuchen ungefährunver- }\end{array}$ \\
\hline $\begin{array}{l}\text { Wiederherstellung der drei- } \\
\text { fachen Kommunikation } \\
\text { und des Nulldruckes. }\end{array}$ & 0 & 34,3 & 0,7 & $\begin{array}{l}\text { änderlich durch Wieder- } \\
\text { herstellungder dreifachen } \\
\text { Kommunikation zwischen }\end{array}$ \\
\hline $\begin{array}{l}\text { Kommunikation zwischen } \\
\text { Bürette und Manometer } \\
\text { Kommunikation }\end{array}$ & 5 & 34,7 & - & $\begin{array}{l}\text { dem Hen Hineinlaufen des } \\
\text { Wassers in die Blase. }\end{array}$ \\
\hline $\begin{array}{l}\text { Kommunikation zwischen } \\
\text { Bürette und Harnblase. }\end{array}$ & $\overline{10}$ & $\begin{array}{l}35,3 \\
35,6 \\
36,3\end{array}$ & $\frac{0,6}{0,7}$ & \\
\hline
\end{tabular}


Die Harnblase als Expulsivorgan. Die glatte Muskeltaser.

\begin{tabular}{|c|c|c|c|c|}
\hline $\begin{array}{c}\text { Vorbereitende } \\
\text { und nachfolgende Akte }\end{array}$ & $\begin{array}{c}\text { Druck } \\
\text { in mm } \\
\mathrm{Hg}\end{array}$ & $\begin{array}{c}\text { Wasser } \\
\text { in der } \\
\text { Bürette } \\
\text { ccm }\end{array}$ & $\begin{array}{c}\text { Wasser } \\
\text { in der } \\
\text { Blase }\end{array}$ & Bemerkungen \\
\hline & $\begin{array}{l}\frac{15}{20} \\
\frac{-}{25} \\
\frac{30}{30} \\
\frac{35}{40} \\
\frac{45}{50} \\
\frac{50}{-}\end{array}$ & $\begin{array}{l}36,6 \\
37,3 \\
37,7 \\
38,2 \\
38,6 \\
39,1 \\
39,4 \\
39,8 \\
40,2 \\
40,6 \\
41,0 \\
41,3 \\
41,7 \\
42,0 \\
42,4 \\
42,6\end{array}$ & $\begin{array}{l}\overline{0,7} \\
\overline{0,5} \\
\overline{0,5} \\
\overline{0,4} \\
\overline{0,4} \\
\overline{0,3} \\
\overline{0,3} \\
\overline{0,3} \\
\overline{0,2}\end{array}$ & $\begin{array}{l}\text { Die Harnblase transsudiert } \\
\text { an einzelnen Stellen. } \\
\text { Die Harnblase transsudiert } \\
\text { an mehreren Punkten. } \\
\text { Die ganze Harnblase trans- } \\
\text { sudiert, dabei ist auch die } \\
\text { Ebene } p \text { (Fig. 2) befeuch- } \\
\text { tet. } \\
\text { DieHarnblase trieft deutlich. }\end{array}$ \\
\hline
\end{tabular}

Tabelle IV.

Exstirpierte Meerschweinchen-Harnblase (ㅇ).

\begin{tabular}{|c|c|c|c|c|}
\hline $\begin{array}{l}\text { Vorbereitende } \\
\text { und nachfolgende Akte }\end{array}$ & $\begin{array}{c}\text { Druck } \\
\text { in } \mathrm{mm} \\
\mathrm{Hg}\end{array}$ & $\begin{array}{l}\text { Wasser } \\
\text { in der } \\
\text { Burette } \\
\text { ccm }\end{array}$ & $\begin{array}{c}\text { Wasser } \\
\text { in der } \\
\text { Harn- } \\
\text { blase }\end{array}$ & Bemerkungen \\
\hline $\begin{array}{l}\text { Nivellierung und Fullung } \\
\text { des ganzen Systems mit } \\
\text { Wasser. Hahn in drei- } \\
\text { facher Kommunikation } \\
\text { und Aufsuchung des Null- } \\
\text { druckes. - . - . } \\
\text { Kommunikation zwischen } \\
\text { Bürette und Harnblase; } \\
\text { Entleerung der Blase - } \\
\text { Wiederherstellung der drei- } \\
\text { fachen Kommunikation } \\
\text { und des Nulldruckes. } \\
\text { Kommunikation zwischen } \\
\text { Bürette und Manometer. } \\
\text { Kommunikation zwischen } \\
\text { Bürette und Harnblase. }\end{array}$ & $\begin{array}{l}\frac{-}{10} \\
\frac{15}{-} \\
\frac{20}{35} \\
\frac{30}{35} \\
\frac{35}{-}\end{array}$ & $\begin{array}{l}87,6 \\
\\
89,8 \\
90,2 \\
90,9 \\
91,3 \\
91,8 \\
92,2 \\
92,5 \\
92,8 \\
93,1 \\
93,5 \\
93,7 \\
94,2 \\
94,4\end{array}$ & $\begin{array}{l}- \\
2,2 \\
\overline{0,7} \\
\overline{0,5} \\
\overline{0,3} \\
\overline{0,3} \\
\overline{0,2} \\
\overline{0,2}\end{array}$ & $\begin{array}{l}\text { Ungefähre, für jede zwei- } \\
\text { malige Ablesung (Mano- } \\
\text { meter, Bürette) verwen- } \\
\text { dete Zeit }=1 \text { Min. } \\
\text { Wassertemperatur }=12^{\circ} \mathrm{C} \text {. } \\
\text { So gut wie unveränderlich } \\
\text { sind die Drucke in den } \\
\text { Kontrollversuchen durch } \\
\text { Wiederherstellung der } \\
\text { dreifachen Kommuni- } \\
\text { kation zwischen einem } \\
\text { Sinken des Wassers in } \\
\text { die Harnblase und dem } \\
\text { anderen. }\end{array}$ \\
\hline
\end{tabular}


Tabelle V.

Exstirpierte Harnblase eines grossen Meerschweinchens (む).

\begin{tabular}{|c|c|c|c|c|}
\hline $\begin{array}{c}\text { Vorbereitende } \\
\text { und nachfolgende Akte }\end{array}$ & $\begin{array}{c}\text { Druck } \\
\text { in } \mathrm{mm} \\
\mathrm{Hg}\end{array}$ & $\begin{array}{c}\text { Wasser } \\
\text { in der } \\
\text { Bürette } \\
\mathrm{ccm}\end{array}$ & $\begin{array}{c}\text { Wasser } \\
\text { in der } \\
\text { Harn- } \\
\text { blase }\end{array}$ & Bemerkungen \\
\hline $\begin{array}{l}\text { Nivellierung und Füllung } \\
\text { des ganzen Systems mit } \\
\text { Wasser. Hahn in drei- } \\
\text { facher Kommunikation } \\
\text { und Aufsuchung des Null- } \\
\text { durckes. . . . . } \\
\text { Kommunikation zwischen } \\
\text { Bürette und Manometer, } \\
\text { dann zwischen Bürette } \\
\text { und Harnblpse. . . . . }\end{array}$ & $\begin{array}{r}5 \\
10 \\
15 \\
20 \\
30 \\
40 \\
\frac{50}{50} \\
\frac{60}{7} \\
\frac{70}{50} \\
\frac{80}{90}\end{array}$ & $\begin{array}{c}22,3 \\
22,7 \\
23,1 \\
23,5 \\
24,3 \\
25,0 \\
25,3 \\
26,0 \\
26,3 \\
27,1 \\
27,4 \\
28,1 \\
28,4 \\
29,1 \\
29,4 \\
-\end{array}$ & $\begin{array}{l}- \\
\overline{-} \\
- \\
- \\
\overline{0,3} \\
\overline{0,3} \\
\overline{0,3} \\
\overline{0,3} \\
\overline{0,3} \\
\overline{0,3} \\
\overline{0,3}\end{array}$ & $\begin{array}{l}\text { Gleich nach Exstirpation } \\
\text { der Harnblase mitsamt } \\
\text { der Prostata, den Testi- } \\
\text { keln und den Nieren } \\
\text { wird die Kanüle bis } \\
\text { nahe an den äusseren } \\
\text { Sphinkter eingefúhrt, ohne } \\
\text { ihn jedoch zu passieren. } \\
\text { Zu jeder zweimaligen Ab- } \\
\text { lesung ungefähr nötige } \\
\text { Zeit(Manometer, Bürette) } \\
=1 \text { Min. Wassertempera- } \\
\text { tur =12 } \mathrm{C} \text {. Drucke un- } \\
\text { veränderlich (I). }\end{array}$ \\
\hline
\end{tabular}

Tabelle VI.

Exstirpierte Harnblase von einem kleinen Meerschweinchen ( $(t)$.

\begin{tabular}{|c|c|c|c|c|}
\hline $\begin{array}{l}\text { Vorhereitende } \\
\text { und nachfolgende Akte }\end{array}$ & $\begin{array}{c}\text { Druck } \\
\text { in mm } \\
\mathrm{Hg}\end{array}$ & $\begin{array}{c}\text { Wasser } \\
\text { in der } \\
\text { Bürette } \\
\text { ccm }\end{array}$ & $\begin{array}{l}\text { Wasser } \\
\text { in der } \\
\text { Harn- } \\
\text { blase }\end{array}$ & Bemerkungen \\
\hline $\begin{array}{l}\text { Nivellierung und Fullung } \\
\text { des ganzen Systems mit } \\
\text { Wasser. Hahn in drei- } \\
\text { facher Kommunikation } \\
\text { und Aufsuchung des Null- } \\
\text { druckes. . . . . . . . }\end{array}$ & 0 & 38,8 & - & $\begin{array}{l}\text { Gleich nach Exstirpierung } \\
\text { der Harnblase mitsamt } \\
\text { der Prostata, den Hoden } \\
\text { und den Nieren wird die } \\
\text { Kanüle eingeführt unter } \\
\text { Überwindung des änsseren } \\
\text { Sphinkters. }\end{array}$ \\
\hline
\end{tabular}




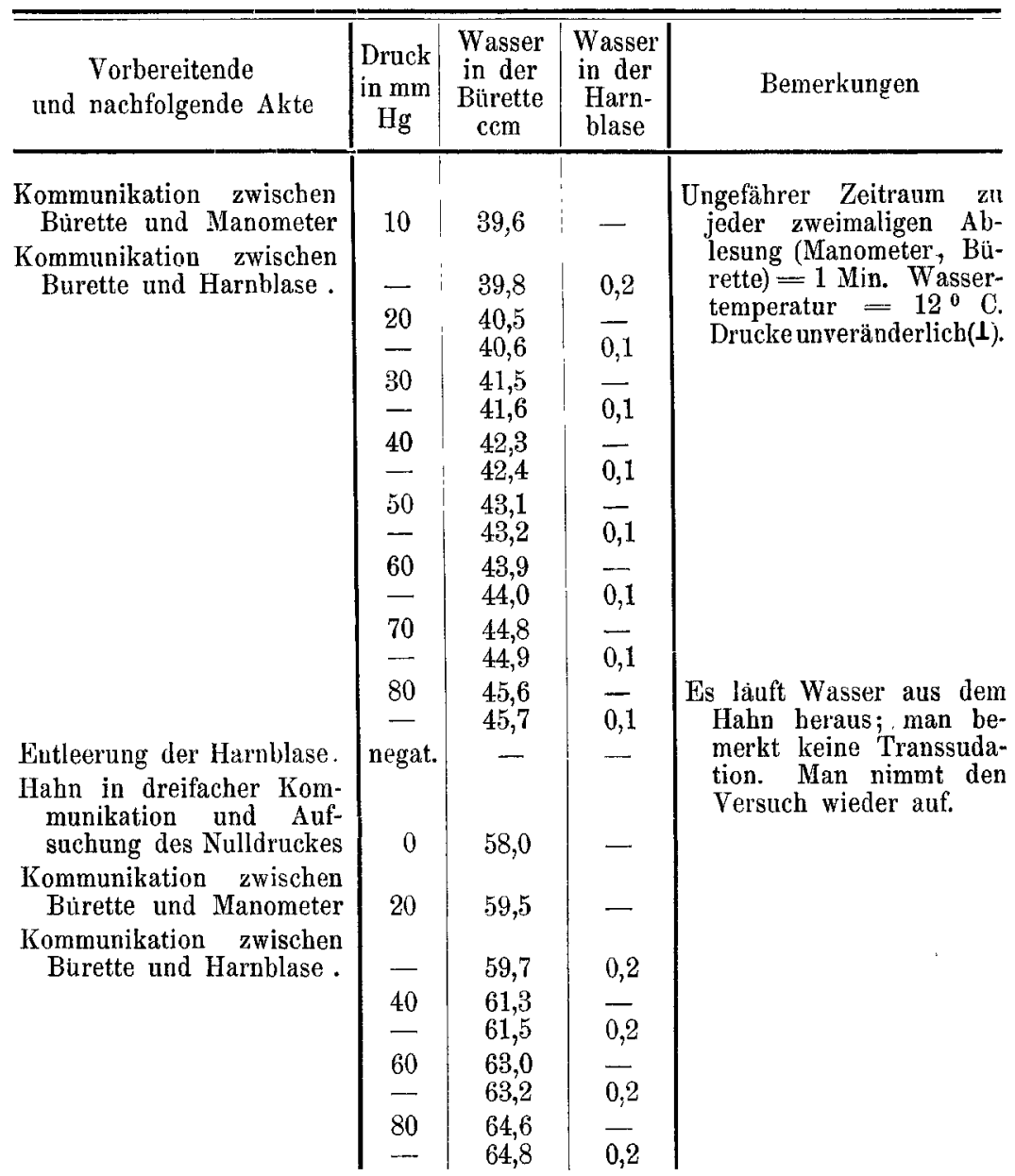

\section{Transsudation aus Harnblasen infolge von unter Druck enthaltener Flüssigkeit. Elastizität der lebenden, mit zirku- lierendem Blut versorgten Harnblase.}

Wir haben bereits bei der veränderten Harnblase des Meerschweinehens auf die Transsudation hingewiesen, die schon bei 30-35 mm Druck bedeutend ist. Diese Transsudation ist auf der äusseren Blasenoberfläche an wenigen zerstreuten Tröpfchen erkennbar, die kleinen Schweisstropfen vergleichbar sind; in der Folge werden die Tröpfchen zahlreich, nähern sich und fliessen zu grossen Tropfen zusammen, wodurch der Prozess den Anschein einer echten Filtration gewinnt. 
Da nach Entledigung der Blase von der in ihr enthaltenen Flüssigkeit und nach deren Ausdrücken zwischen den Fingern dieselbe noch wie ein kleiner Schwamm weitertropft, kann es keinem Zweifel obliegen, dass Wasser in ihrem Gewebe zurückbehalten worden ist unter Verschluss der Poren, die durch den aus dem Innern ausgeübteu Druck erweitert worden waren; dies stellt eben die Imbibition dar.

Wie soll man sich jedoch die Tatsache erklären, dass es Blasen gibt, die bei $30-35 \mathrm{~mm}$ tatsächlich filtrieren, während es anderseits solche gibt, die bei $80 \mathrm{~mm}$ Druck gar keine Spur von Transsudation aufweisen? Man wird vielleicht darauf antworten, dass die letzteren sehr wenig Wasser aufnahmen, während die anderen davon mehrere Kubikzentimeter erhielten. Diese Annahme verliert jedoch jeglichen Wert, sobald man bedenkt, dass die lebende Harnblase, d. h. bei zirkulierendem Blute, sich durch viel grössere Flüssigkeitsvolumina ausdehnen lässt, ohne dass ein Tröpfchen Wasser an die äussere Oberfläche gelangt.

Man wird einwenden, dass die in den erwähnten Versuchen benützten filtrierenden Harnblasen sicherlich etwas von ihrer Elastizität eingebüsst hatten. Diese ungefähre Beurteilung kann jedoch denjenigen nicht befriedigt lassen, der in strenger Weise experimentieren will, und ich hielt es daher für notwendig, die einzelnen Teilakte der angestellten Untersuchungen in genau abschätzbaren graphischen Bildern darzulegen.

Die Sache musste leicht gelingen; es genügte schon, einen kurzen, hohlen Glaszylinder zu nehmen, der an den Enden mit eingeschliffenen Stöpseln verschlossen ist, die ihrerseits zwei Metallröhrehen tragen, ein zuführendes für das Organ, das daran angebunden wird, und ein abführendes für die Schreibkapsel. Wenn man das zuführende Röhrchen mit dem Ast $c$ (Fig. 2) und die Wanne des grossen Manometers mit einer anderen analogen Schreibkapsel verbindet, können die schreibenden Federn in doppelter Aufzeichnung die einzelnen vorhin erwähnten Akte festlegen. Die Aufzeichnung auf Fig. 5 erhielt man mit der Gummiblase aus der Tab. I; sie weicht wenig von derjenigen ab, die man erhalten hätte, wenn man die Harnblase auf Tab. V und VI benützt hätte.

$a b$ der Fig. 5 bedeutet die Linie, nachdem man eine der Schreibkapseln mit dem Metallmanometer Marey's in Verbindung gesetzt und 
in diesem einen Druck von $20 \mathrm{~mm}$ erzeugt hat. Der dreiwegige Hahn (Fig. 2) war derart gedreht, dass die Blase ausgeschaltet wurde.

$a^{\prime} b^{\prime}$ bedeutet die mit der anderen Schreibkapsel erhaltene Linie (Blase bei Nulldruck):

$b c$ ist die absteigende Linie des Manometerdrucks. Der Hahn wurde so gedreht, dass die Bürette ausgeschaltet wurde.

$b^{\prime} c^{\prime}$ bedeutet die Linie der gleichzeitigen Aufbläbung der Blase durch Wasser, das aus der Manometerkapsel in sie eindringt.

$d^{\prime} e^{\prime}$ bedentet die Kurve der zweiten Aufblähung der Blase durch Wasser, das aus der Bürette kommt, sobald der Hahn so gedreht wird, dass eine Kommunikation zwischen ihnen hergestellt wird.

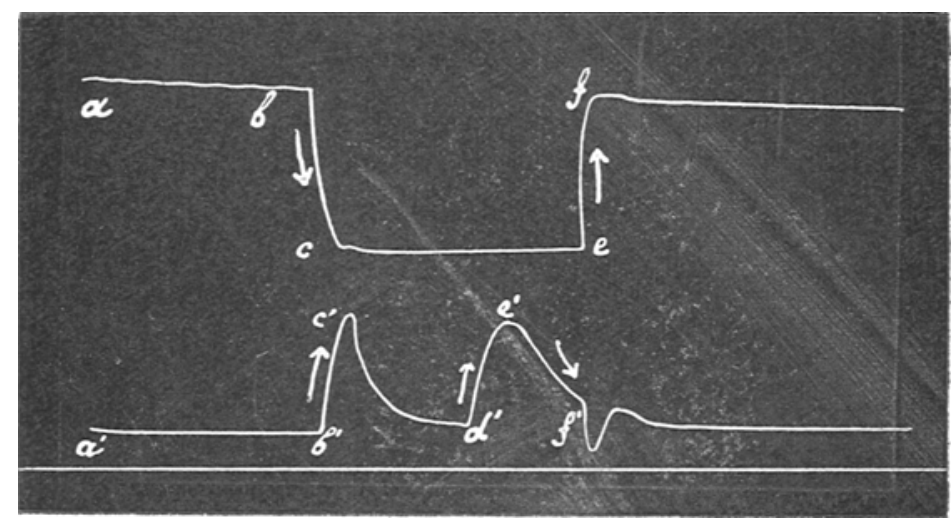

Fig. 5.

$e^{\prime} f^{\prime}$ bedeutet die Kurve der elastischen Reaktion der Blase.

ef bedeutet die Linie des wiederaufsteigenden Manometerdrucks, sobald die drei Wege der Röhre $a b c$ in Funktion treten (Fig. 2). Die Aufzeichnung auf der Fig. 6 erhielt man dagegen mit der Gummiblase aus Tab. II; sie weicht jedoch von derjenigen ab, die man hätte bekommen können, wenn man die Harnblase aus Tab. III und IV verwendet hätte. Bei einem gewissen Punkt (30 mm Hg) uberdehnte sich die Gummiblase unter bedeutender Abnahme des Anfangsdrucks; die Linie $f g$ steigt in der Tat deutlich herunter.

Derselbe Versuch, mit den transsudierenden Harnblasen (35 bis $40 \mathrm{~mm} \mathrm{Hg}$ ) aus den Tab. III und IV angestellt, ergab ähnliche Aufzeichnungen, jedoch mit fast horizontalen Drucklinien $(f g)$, und zwar in vollkommener Übereinstimmung mit dem, was auf denselben Tabellen unter "Bemerkungen" geschrieben steht. 
Es ist nicht ohne Wert, beizufügen, dass bei denselben Druckhöhen $(35-40 \mathrm{~mm} \mathrm{Hg}$ ) aus solchen Blasen hypotonische, isotonische, hypertonische Kochsalzlösungen, die Ring er'sche Lösung sowie die Kuhmilch mit ihren kleinsten Fettkügelchen $(0,2-0,5 \mu)$ durchfiltrierten.

Es wäre sicherlich interessant, das Verhalten der verschiedenen Lösungen in Harnblasen zu untersuchen, in denen noch die volle oder nur wenig verminderte kontraktile Funktion besteht, und noch angebrachter wäre es, identische Versuche an normal mit Blut versorgten Blasen vorzunehmen.

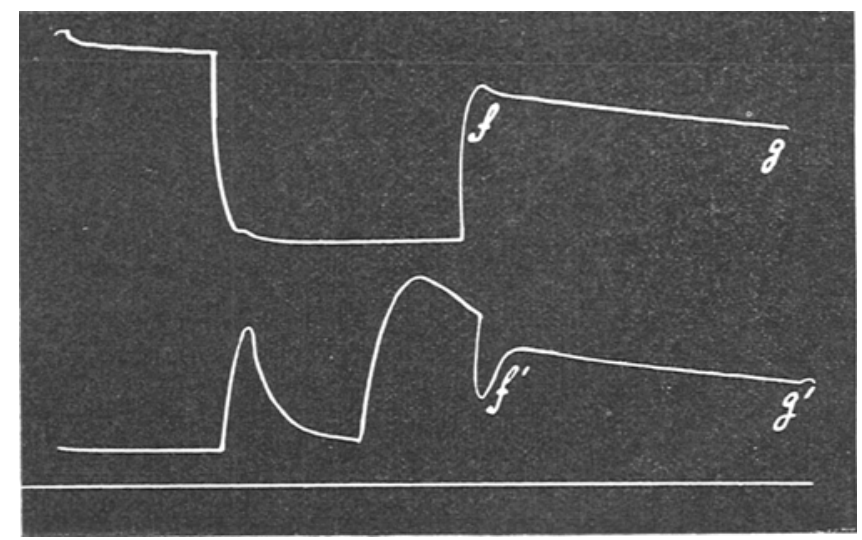

Fig. 6.

Diese Untersuchungen über die Imbibition und die Transsudation hätte man mit denen von $\mathrm{Fasola}$ und Galeotti ${ }^{1}$ ) üher die Permeabilität verbinden können; wir haben jedoch unterlassen, näher darauf einzugehen, um das Thema nicht aus dem Auge zu verlieren, das wir uns eigentlich vorsteckten und das die komplizierte Funktion der Blase betrifft.

Zusammenfassend ist es angebracht, an das verschiedene Verhalten der Elastizität zu erinnern, wie es sich in seit wenigen Minuten herausgeschnittenen Blasen darstellt (Durchschneiden der Halsgefässe, Enthauptung) und in solchen, die nach einigen Stunden entfernt werden. In den ersteren war die Elastizität vollkommen, in den letzteren unvollkommen; in diesen fand man die Phasen der

1) G. Fasola et G. Galeotti, Recherches expérimentales sur la perméabilité de la vessie. Arch. ital. de Biol. t. 39 p. 292-295. 1903. 
Auseinanderfaltung, der Debnung und elastischen Reaktion, der Imbibition und Transsudation, in jenen nur die Zwischensphase und unter deutlich regelmässigem Verlauf.

Dies alles würde jedoch noch nicht genügen, um verständlich zu machen, in welcher Weise sich unter den bekannten Versuchsbedingungen die. Blase in situ, mit ihren normalen Beziehungen, und gehörig mit Blut versorgt verhalten hätte. Wir wollen hier den Leser nicht mit der detaillierten Beschreibung vieler in dieser Beziehung ausgeführten Untersuchungen langweilen, und fassen wir besser die versehiedenen Ergebnisse folgendermaassen zusammen:

„Die lebende Harnblase zeigt in situ und mit zirkulierendem Blut versorgt ein verschiedenes Verhalten ihrer Elastizität; im Beginn jeder Untersuchung ist sie wenig, in den Zwischenstadien sehr elastisch; sie weist die Phasen der Auseinanderfaltung, der Dehnung und der elastischen Reaktion auf und nur ausnahmsweise (bei Druckhöhen, die in vivo nicht in Frage kommen) auch die Phase der Imbibition und der Transsudation.

„Selten lässt sich auch mit dem bis zur Blaseneinmündung eingeführten Katheter Urin entleeren, den sie auf Grund eines starken, durch das Sinken der Bürette bedingten negativen Druckes enthält; entsprechende Manipulationen auf dem Hypogastrium erleichtern immer das Ergebnis. Dies ist bei den männlichen Meerschweinchen gewöhnlich der Fall; bei den weiblichen Tieren folgt auf die Einführung des Katheters gewöhnlich Harn- und Kotentleerung.

„Nach Entleerung der Blase und Wiederherstellung des manometrischen Nulldrucks nimmt das Organ Wasser auf, falls es zusammengefallen ist; dagegen nimmt es keines auf, wenn es sich in aktiver Kontraktionsstellung befindet. Wenn der Katheter sich an der Blaseneinmündung befindet, genügt meist ein Druck von $5-10 \mathrm{~mm} \mathrm{Hg}$, um jenen Widerstand zu überwinden und Flüssigkeit in bedeutender Menge eindringen zu lassen, besonders wenn es auf $36-38^{\circ} \mathrm{C}$. erwärmt ist.

„Diese erste Wassermenge ist genau proportional mit der Weite des Organs, das gedehnt werden soll. Während der Dehnung sinkt der anfängliche manometrische Druck im Augenblick, in welchem der Hahn von der Stellung $\rightarrow$ (Verbindung zwischen Blase und Bürette) in die Stellung $\perp$ (Verbindung zwischen Bürette, Blase und Manometer) gebracht wird.

"Wenn der Anfangsdruck mit dem in dreifacher Verbindung 
stehenden Hahn sich zu erhalten sucht, kommen die elastische Reaktion sowie die Zusammenziehung des Detrusors zur Geltung. „Wenn man sie kombiniert, beobachtet man starke Niveauschwankungen in der Bürette und im Manometer.

"Wenn die elastische Reaktion allein zur Wirkung kommt, erhält man Gleichgewicht des Manometerdrucks, d. h., wenn man einen Druck von z. B. $20 \mathrm{~mm}$ hergestellt hat (Verbindung zwischen Bürette und Manometer) und z. B. um 0,5-2 cem heruntergeht (Verbindung zwischen Bürette und Blase), ändert sich das Niveau des Manometers nicht, auch wenn die dreifache Verbindung wiederhergestellt wird.

"Von diesem Momente an werden die eingelassenen Mengen mit prompten und proportionalen elastiscben Reaktionen im Verhältnis zur erhöhten Resistenz der Blasenwandungen immer kleiner. Die Volumablesungen werden mit grosser Schnelligkeit und Genauigkeit ausgefülıtt, natürlich vorausgesetzt, dass keine Muskelkontraktionen stattfinden.

"Sobald die Ablesung an der Bürette schwieriger und langsamer erfolgt, pflegt ein leichtes Überwiegen der Dehnbarkeit über die clastische Reaktion einzutreten.

"Wenn man annimmt, dass die Narkose des Tieres weiter dauert, utd wenn man von den aktiven und den passiven Blasenkontraktionen absieht, dann wird man in die Blase variable Flüssigkeitsvolumina eintreten sehen; dabei rufen die wegen des Inhaltes eintretenden Organdeformationen immer noch bedeutende elastische Reaktionen hervor.

"Während es bei den dünnen und dehnbaren Gummiblasen (Tab. II) möglich ist, mit einem gewissen Druck die Elastizitätsgrenze zu überwinden und so eine Überdehnung zu bewirken, findet díes in den Harnblasen nicht statt, da sie so viel Flüssigkeit in sich aufuehmen, als dieselbe sie in den höheren Lagen der Bürette dàzu zwingt. Natürlich blähen sie sich deswegen auf; ihre Wandungen werden immer dünner, die Gefässwandungen verschliessen sich, die Zirkulation wird total unterdrückt, die Parenchymporen erweitern sich, und ès beginnt die pärtielle Transsudation.

„Endlich kommt es, sofern man den Druck nicht sofort erniedrigt und das Organ teilweise oder gänzlich entleert, zur Filtration, wodurch dessen Vitalität für immer in Frage gestellt wird." 